\title{
Amiodarone-induced Thyrotoxicosis with Cardiopulmonary Arrest
}

\author{
Kosuke Inoue ${ }^{1}$, Jun Saito ${ }^{1}$, Tetsuo Kondo ${ }^{2}$, Kaoru Miki ${ }^{3}$, Chiho Sugisawa ${ }^{1}$, Yuya Tsurutani ${ }^{1}$, \\ Naoki Hasegawa ${ }^{4}$, Shinya Kowase ${ }^{5}$, Yukio Kakuta ${ }^{4}$, Masao Omura ${ }^{1}$ and Tetsuo Nishikawa ${ }^{1}$
}

\begin{abstract}
:
We describe a case of amiodarone-induced thyrotoxicosis (AIT) with cardiopulmonary arrest (CPA) in a 49-year-old woman. The patient had been treated with amiodarone for non-sustained ventricular tachycardia. Two weeks prior to her admission, she developed thyrotoxicosis and prednisolone (PSL, $30 \mathrm{mg}$ daily) was administered with the continuation of amiodarone. However, she was admitted to our hospital for CPA. We performed total thyroidectomy to control her thyrotoxicosis and the pathological findings were consistent with type 2 AIT. She gradually improved and was discharged on day 84. This case demonstrates the importance of considering immediate total thyroidectomy for patients with uncontrollable AIT.
\end{abstract}

Key words: amiodarone-induced thyrotoxicosis, hyperthyroxinemia, cardiopulmonary arrest, prednisolone, thyroidectomy

(Intern Med 57: 59-63, 2018)

(DOI: 10.2169/internalmedicine.9177-17)

\section{Introduction}

Amiodarone, a widely used antiarrhythmic drug, is known to cause thyroid dysfunction, such as amiodarone-induced thyrotoxicosis (AIT) and amiodarone-induced hypothyroidism (AIH) through its pharmacological and toxic actions $(1,2)$. AIT represents a diagnostic and therapeutic challenge, while AIH does not cause critical problems in most cases. AIT is classified as type 1 (iodine-induced hyperthyroidism), type 2 (destructive thyroiditis), and mixed type (types 1 and 2) (3). In Japan, where the iodine intake is sufficient, almost all cases of AIT are type 2 and are mainly treated with prednisolone (PSL) (4). However, some cases of type 2 AIT are reported to be resistant to oral PSL therapy $(5,6)$. Moreover, patients who are treated with amiodarone usually have severe cardiac abnormalities and amiodarone withdrawal is often difficult. In such cases, immediate total thyroidectomy should be considered as a second-line therapy (3) because prolonged thyrotoxicosis has the poten- tial to cause cardiac damage and muscle weakness (7). We herein report a case of uncontrolled type 2 AIT with cardiopulmonary arrest (CPA) which ultimately required total thyroidectomy.

\section{Case Report}

A 49-year-old woman was referred to our hospital for thyrotoxicosis. Her significant medical history included severe hypertrophic cardiomyopathy and non-sustained ventricular tachycardia (NSVT). She had been treated with amiodarone (100 $\mathrm{mg}$ per day) and an implantable cardioverter-defibrillator (ICD) for 2 years prior to her presentation. She had no personal or family history of thyroid disease. After the initiation of amiodarone treatment, her free thyroxine (FT4), free triiodothyronine (FT3) and thyroid-stimulating hormone (TSH) levels were measured at 3 -month intervals. The levels remained normal for the first 2 years of the therapy; however, her most recent test showed hyperthyroxinemia with elevated FT4 (>7.8 ng/dL) and FT3

\footnotetext{
${ }^{1}$ Endocrinology and Diabetes Center, Yokohama Rosai Hospital, Japan, ${ }^{2}$ Department of Human Pathology, University of Yamanashi Interdisciplinary Graduate School of Medicine and Engineering, Japan, ${ }^{3}$ Departments of Endocrinology and Diabetes, JCHO Tokyo Yamate Medical Center, Japan, ${ }^{4}$ Department of Pathology, Yokohama Rosai Hospital, Japan and ${ }^{5}$ Department of Cardiovascular Medicine, Yokohama Rosai Hospital, Japan
}

Received: March 11, 2017; Accepted: April 19, 2017; Advance Publication by J-STAGE: October 16, 2017

Correspondence to Dr. Jun Saito, saitoj@yokohamah.johas.go.jp 
Table. Laboratory Findings.

\begin{tabular}{|c|c|c|c|c|c|}
\hline \multicolumn{4}{|c|}{ Blood samples } & \multicolumn{2}{|c|}{ Thyroid function } \\
\hline WBC & $1.93 \times 10^{4} / \mu \mathrm{L}$ & $\mathrm{TP}$ & $6.7 \mathrm{~g} / \mathrm{dL}$ & TSH & $<0.005 \mu \mathrm{IU} / \mathrm{mL}$ \\
\hline $\mathrm{Hb}$ & $12.7 \mathrm{~g} / \mathrm{dL}$ & Alb & $3.7 \mathrm{~g} / \mathrm{dL}$ & Free T4 & $>7.8 \mathrm{ng} / \mathrm{dL}$ \\
\hline Plt & $28.4 \times 10^{4} / \mu \mathrm{L}$ & $\mathrm{Na}$ & $138 \mathrm{mEq} / \mathrm{L}$ & Free $\mathrm{T} 3$ & $19.2 \mathrm{pg} / \mathrm{mL}$ \\
\hline AST & $141 \mathrm{IU} / \mathrm{L}$ & K & $5.6 \mathrm{mEq} / \mathrm{L}$ & Tyroglobulin & $173.4 \mathrm{ng} / \mathrm{mL}$ \\
\hline ALT & $97 \mathrm{IU} / \mathrm{L}$ & $\mathrm{P}$ & $3.9 \mathrm{mg} / \mathrm{dL}$ & TRAb $\left(3^{\text {rd }}\right)$ & $<0.4$ IU/L \\
\hline ALP & $435 \mathrm{IU} / \mathrm{L}$ & $\mathrm{Ca}$ & $9.8 \mathrm{mg} / \mathrm{dL}$ & TPOAb & $<0.3 \mathrm{IU} / \mathrm{L}$ \\
\hline LDH & $326 \mathrm{IU} / \mathrm{L}$ & Glucose & $227 \mathrm{mg} / \mathrm{dL}$ & $\mathrm{TgAb}$ & $1.6 \mathrm{IU} / \mathrm{L}$ \\
\hline $\mathrm{CK}$ & $55 \mathrm{IU} / \mathrm{L}$ & $\mathrm{BNP}$ & $208.1 \mathrm{pg} / \mathrm{mL}$ & & \\
\hline BUN & $46.3 \mathrm{mg} / \mathrm{dL}$ & KL-6 & $156 \mathrm{IU} / \mathrm{mL}$ & & \\
\hline $\mathrm{Cr}$ & $0.68 \mathrm{mg} / \mathrm{dL}$ & CRP & $0.03 \mathrm{mg} / \mathrm{dL}$ & & \\
\hline
\end{tabular}

WBC: white blood cell count, Hb: hemoglobin, Plt: platelet, AST: aspartate aminotransferase, ALT: alanine aminotransferase, ALP: alkaline phosphatase, LDH: lactate dehydrogenase, BUN: blood urea nitrogen, TP: total protein, P: phosphorus, BNP: brain natriuretic peptide, KL-6: sialylated carbohydrate antigen: Krebs von den Lungen-6, CRP: C-reactive protein, TSH: thyroid-stimulating hormone, T4: thyroxine, T3: triiodothyronine, TRAb anti-TSH receptor antibody, TPOAb: anti-thyroperoxidase antibody, $\operatorname{TgAb}$ : anti-thyroglobulin antibody

(19.2 pg/mL) levels, suppressed TSH levels $(<0.005 \mu \mathrm{IU} /$ $\mathrm{mL}$ ), and undetectable autoimmune thyroid antibody levels, including anti-thyroglobulin, anti-thyroperoxidase, and antiTSH receptor antibodies. She complained of a 3-month history of increasing fatigue, loss of appetite, and $7 \mathrm{~kg}$ of unintentional weight loss. Type 2 AIT was highly suspected based on her clinical course and the laboratory findings. Amiodarone was continued because her NSVT was difficult to manage with other antiarrhythmic agents. The administration of PSL [30 mg per day, orally $(0.5 \mathrm{mg} / \mathrm{kg})$ ] was therefore initiated with the continuation of amiodarone. However, 2 weeks later, she was admitted to the intensive care unit of our hospital because of an episode of CPA.

On admission, her height and body weight were $165 \mathrm{~cm}$ and $40 \mathrm{~kg}$, respectively. Her Glasgow Coma Scale (GCS) was E1V1M1, her systolic blood pressure was $80 \mathrm{mmHg}$, and her pulse rate was 100 beats/min. She had a small firm grade 2 goiter (WHO classification). Her ICD recording showed ventricular fibrillation (VF), which was treated by direct current cardioversion $(35 \mathrm{~J})$. Table shows the results of the laboratory tests. She had severe thyrotoxicosis, despite the PSL treatment. Her potassium level was $5.6 \mathrm{mEq} / \mathrm{L}$ and her amiodarone concentration was $134.1 \mathrm{ng} / \mathrm{mL}$. Moreover, the patient had a high carbon dioxide level (139 $\mathrm{mmHg}$, diaphragmatic paralysis, and respiratory muscle weakness, which required mechanical ventilation. Echocardiography showed diffuse hypokinesis (estimated ejection fraction, 40\%) and no asynergy. A thyroid ultrasound showed diffuse goiter [thyroid volume calculated by the ellipsoid formula (8), $43.5 \mathrm{~cm}^{3}$ ] and color flow Doppler sonography (CFDS) revealed a lack of intraparenchymal vascularity (Fig. 1).

Other reasons for thyrotoxicosis, such as subacute thyroiditis, toxic multinodular goiter, were less likely than AIT because she had used amiodarone for 2 years, and because she had no signs of inflammation (Table), and no signs of nodules in her thyroid (Fig. 1). In addition to the regional characteristics of iodine sufficiency in Japan, her autoim- mune thyroid antibody negativity and the CFDS findings strengthened the possibility of type 2 rather than type 1 AIT.

Although the PSL dose was increased to $60 \mathrm{mg}(1.0 \mathrm{mg} /$ $\mathrm{kg}$ ) daily, she remained unconscious and her thyroid function tests continued to show hyperthyroxinemia (FT4>7.8 ng/dL, FT3 $>32.6 \mathrm{pg} / \mathrm{mL}$, and TSH $<0.005 \mu \mathrm{IU} / \mathrm{mL})$. Thus, total thyroidectomy was performed on day 7 after admission. Histologically, most of the thyroid follicles were filled with colloid and lined with flattened follicular epithelial cells. We occasionally found atrophic follicles with fibrosis (Fig. 2a and b) and disrupted follicles with the infiltration of macrophages (Fig. 2c). Cytoplasmic vacuolization, deposits of lipofuscin, and the detachment of follicular epithelial cells were observed in these damaged follicles (Fig. 2d). The lymphocytic infiltration was slight. After total thyroidectomy, her thyroid hormone levels gradually decreased. The PSL dose was tapered and levothyroxine was initiated to maintain a euthyroid status on day 12 after admission. Amiodarone (50 mg daily) was continued to control her NSVT. Her unconsciousness, diaphragmatic paralysis, and respiratory muscle weakness gradually improved and she was removed from the mechanical ventilator on day 14. Following rehabilitation, the patient was finally discharged on the 84th day after admission. Treatment with levothyroxine was continued at a dose of $125 \mu \mathrm{g}$. Her clinical course is shown in Fig. 3.

\section{Discussion}

Amiodarone is a benzofuranic iodine-rich antiarrhythmic drug (class III) that causes thyroid dysfunction in $15-20 \%$ of patients (3). Amiodarone contains $75 \mathrm{mg}$ iodine in each 200 $\mathrm{mg}$ tablet and releases approximately $10 \%$ of the iodine as free iodine each day $(1,9)$, which affects the thyroid hormone metabolism. The prevalence of AIT is $2 \%$ and $12 \%$ in iodine-sufficient and iodine-deficient areas, respectively (10). It can also develop after the withdrawal of amiodarone because the drug has a long half-life of approximately 100 


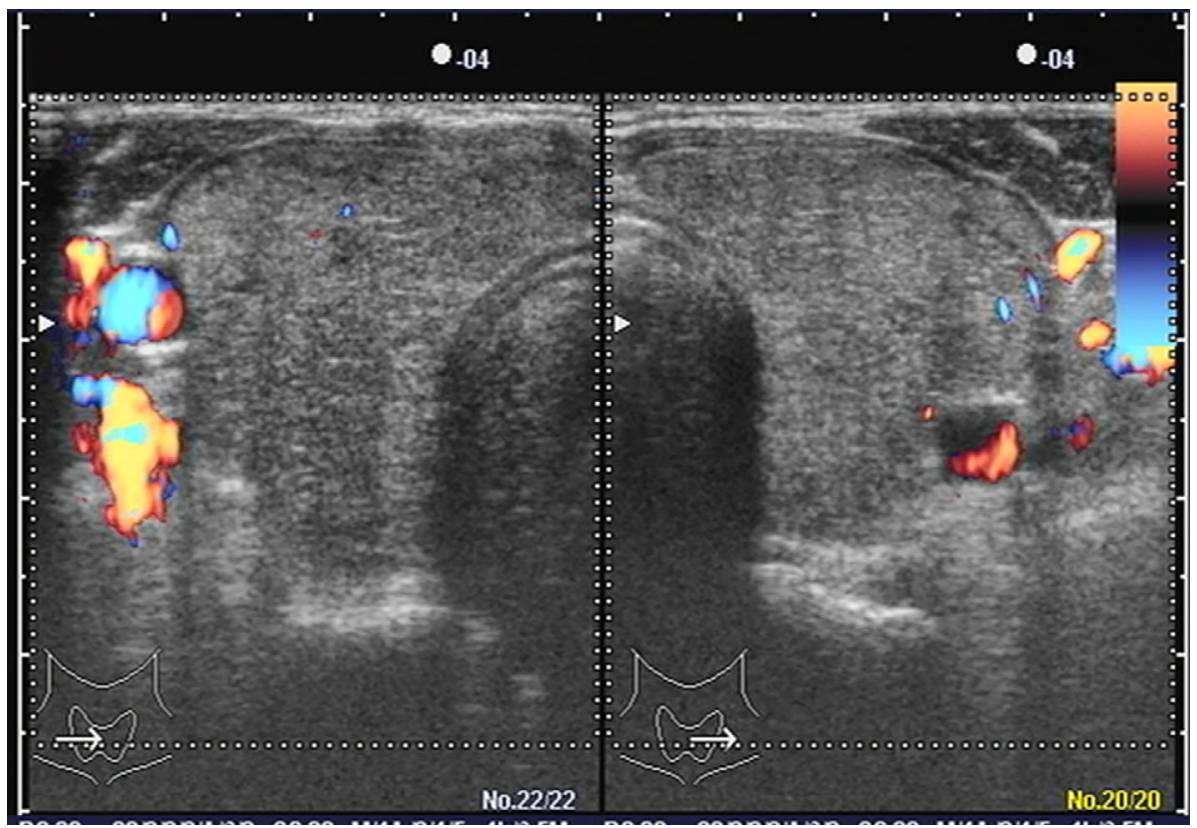

Figure 1. Thyroid ultrasound. Right lobe, 2.2 (wide) $\times 2.7$ (depth) $\times 7.5$ (length) $\mathrm{cm}$; left lobe, $2.1 \times 2.4 \times 7.4 \mathrm{~cm}$; Isthmus, $0.36 \times 3.1 \mathrm{~cm}$. The thyroid volume, as calculated by the ellipsoid formula, was $43.5 \mathrm{~cm}^{3}$. Color flow Doppler sonography revealed no intraparenchymal vascularity.
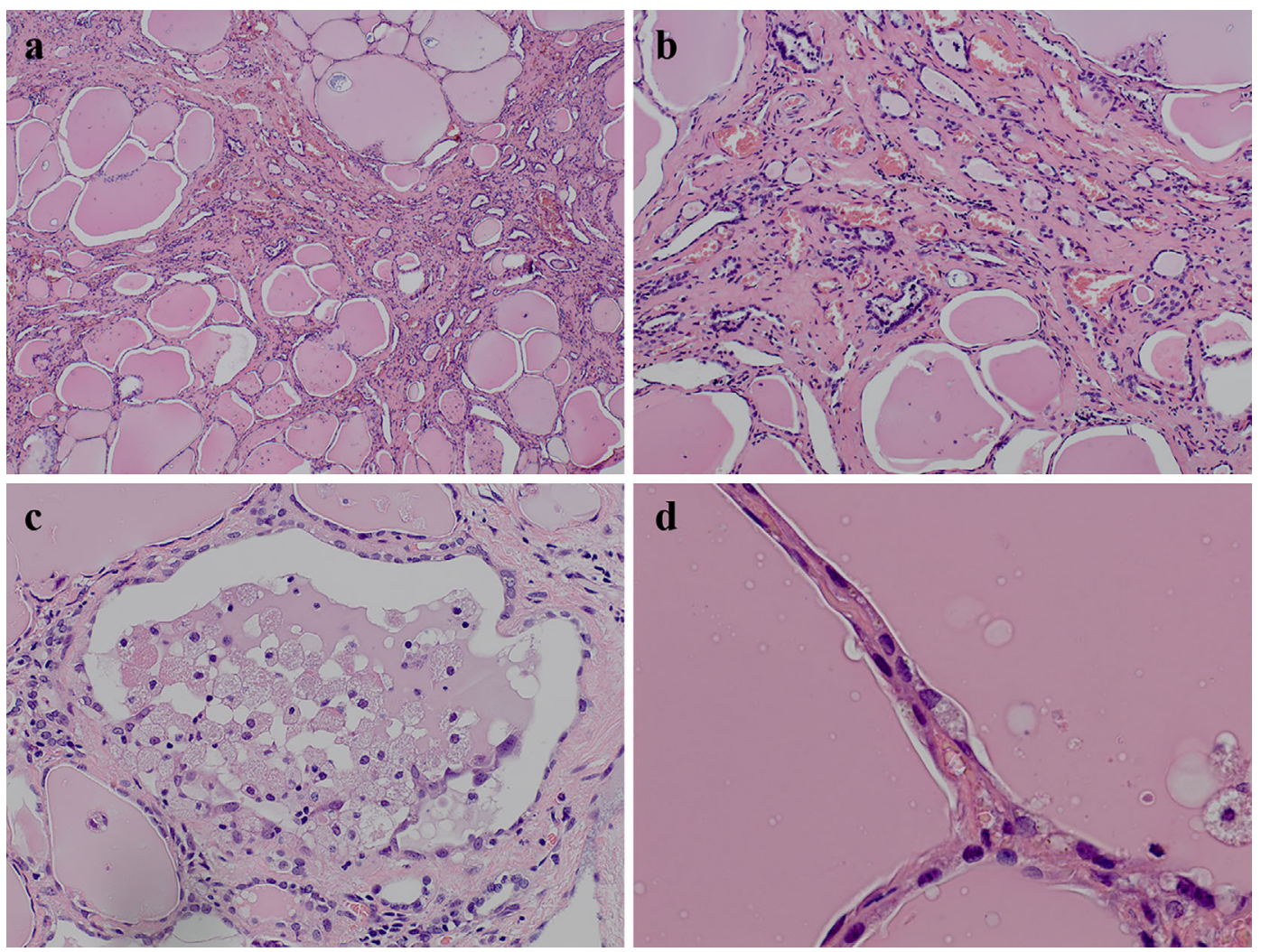

Figure 2. The pathological findings of the thyroid. Atrophic follicles with interstitial fibrosis [a and b: Hematoxylin and Eosin (H\&E) staining]. Disrupted follicles with detachment of the follicular epithelium and the macrophage infiltration (c: H\&E staining). Cytoplasmic vacuolization and lipofuscin in flattened follicular epithelial cells (d: H\&E staining).

days (2). The immediate detection and treatment of AIT are necessary because prolonged thyrotoxicosis causes danger- ous and critical situations for patients with cardiac abnormalities. 


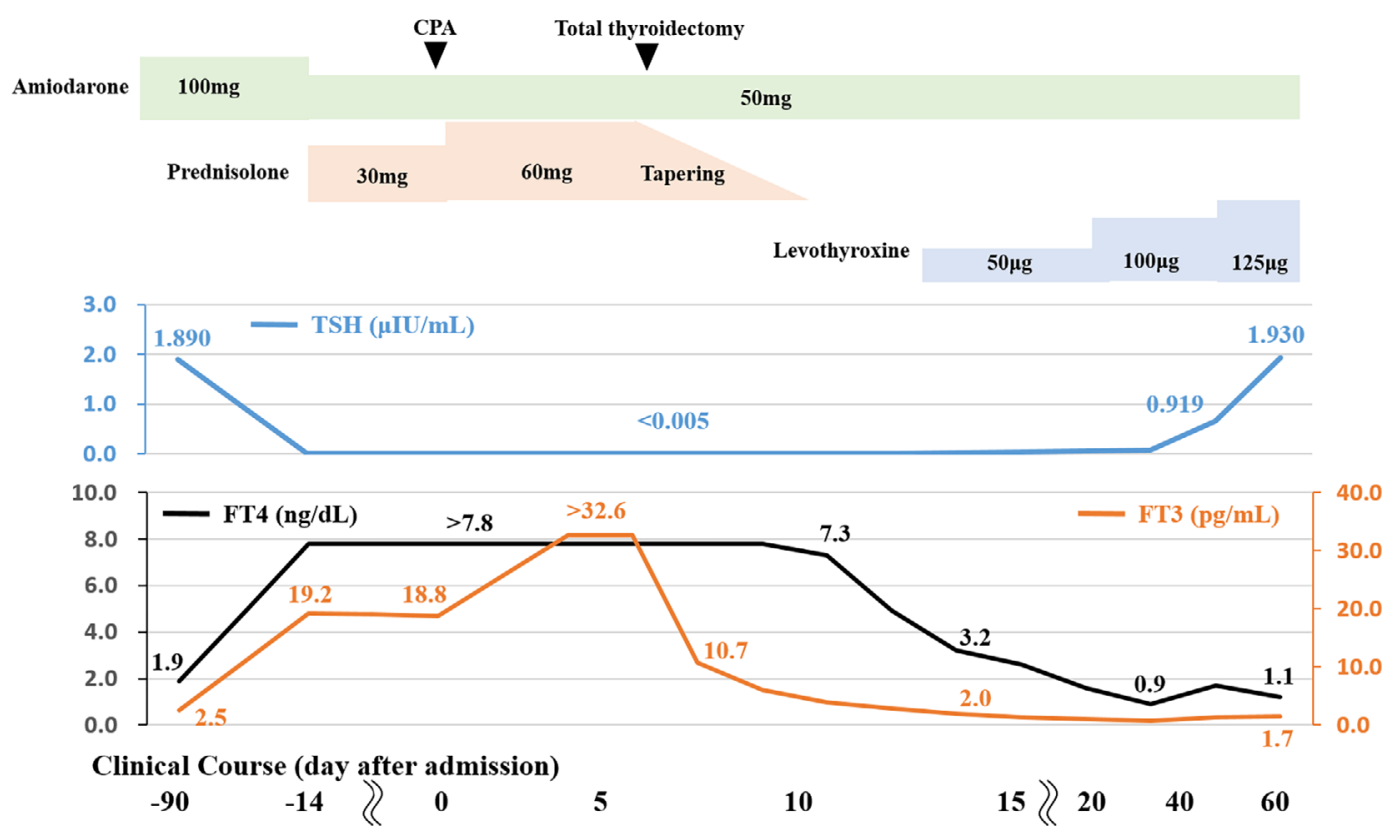

Figure 3. The clinical course. Two weeks after the administration of prednisolone (PSL) for amiodarone-induced thyrotoxicosis, she had an episode of cardiopulmonary arrest (CPA). Total thyroidectomy was performed on day 7 to treat her uncontrolled hyperthyroxinemia. After resection, her thyroid hormone levels gradually decreased. The PSL dose was tapered and levothyroxine was started. Amiodarone was continued to control her non-sustained ventricular tachycardia (NSVT). After rehabilitation, she finally discharged on day 84 .

AIT has two different subtypes: type 1 (a form of iodineinduced hyperthyroidism) and type 2 (a drug-induced destructive thyroiditis) (3). It is important to clarify these two types because they require different therapeutic approaches; antithyroid drugs for type 1 and PSL for type 2 (11). We diagnosed the present case as having type 2 AIT based on the following characteristics: 1) she did not have underlying thyroid disease and was negative for thyroid antibodies; 2) CFDS showed the absence of hypervascularity; and 3) she was living in Japan, where the iodine intake is sufficient and almost all reported AIT cases are type 2 (3). The long duration of amiodarone intake and the increased FT4/FT3 ratio also supported this diagnosis; however, we did not perform a radioactive iodine intake scan or evaluate other parameters such as the serum interleukin-6 level $(5,10)$. We introduced oral PSL as the first-line treatment for type 2 AIT (12), but her thyrotoxicosis was difficult to control with high-dose PSL therapy. A previous study reported that the serum thyroid hormone levels and thyroid volume might predict the response time to PSL in type 2 AIT patients (13). Our patient had high FT4 levels and diffuse goiter, which implied that it would take a significant period of time to achieve a euthyroid status; however, a rapid restoration was needed due to her severe cardiac disease. Thus, we performed total thyroidectomy (14), which led to the improvement of her condition. The pathological findings of the current case were consistent with those of previous reports of amiodaroneinduced destructive thyroiditis $(15,16)$. The destruction of the thyroid follicles, as shown in Fig. 2, would have caused the excessive release of colloid, and may have led to the ele- vated thyroid hormone levels and uncontrollable hyperthyroidism in the present case.

AIT cases with CPA have rarely been described (5). We assumed that her episode of CPA was caused by both cardiac and respiratory dysfunction. Her uncontrollable prolonged thyrotoxicosis might have damaged her cardiac function, which ultimately led to the short-term $\operatorname{VF}(17,18)$. Moreover, the patient had elevated carbon dioxide levels, transient diaphragmatic paralysis, and respiratory muscle weakness. Thyrotoxicosis is well known to cause acuteonset muscle weakness, not only in the distal muscles but also in the proximal and respiratory muscle, through an extracellular shift of intracellular potassium through the increased activity of the $\mathrm{Na}^{+} / \mathrm{K}^{+}$ATP pump (19), which represents a life-threatening condition $(20,21)$. Although the present case did not show hypokalaemia or hypophosphatemia, such electrolyte abnormalities are not necessary to induce myopathy in patients with thyrotoxicosis (7). It is also possible that her emaciation exacerbated her muscle weakness.

In conclusion, we experienced a rare case of type 2 AIT in a Japanese patient with CPA who was completely cured after total thyroidectomy. This case demonstrated the importance of detecting thyroid abnormalities in patients with a history of amiodarone use and of treating them as soon as possible because prolonged thyrotoxicosis might cause CPA. Total thyroidectomy should be considered when patients require the immediate restoration of euthyroidism because of severe conditions.

The authors state that they have no Conflict of Interest (COI). 


\section{References}

1. Basaria S, Cooper DS. Amiodarone and the thyroid. Am J Med 118: 706-714, 2005.

2. Martino E, Bartalena L, Bogazzi F, Braverman LE. The effects of amiodarone on the thyroid. Endocr Rev 22: 240-254, 2001.

3. Bogazzi F, Bartalena L, Martino E. Approach to the patient with amiodarone-induced thyrotoxicosis. J Clin Endocrinol Metab 95: 2529-2535, 2010

4. Uchida T, Kasai T. Prevalence of amiodarone-induced thyrotoxicosis and associated risk factors in Japanese patients. 2014: 534904, 2014.

5. Mehta AN, Vallera RD, Tate CR, Sager RA, Welch BJ. Total thyroidectomy for medically refractory amiodarone-induced thyrotoxicosis. Bayl Univ Med Cent 21: 382-385, 2008.

6. Hashimoto K, Ota M, Irie T, et al. A case of type 2 amiodaroneinduced thyrotoxicosis that underwent total thyroidectomy under high-dose steroid administration. Case Rep Endocrinol 2015: 416145, 2015.

7. Lin SH. Thyrotoxic periodic paralysis. Mayo Clin Proc 80: 99105, 2005.

8. Trimboli P, Ruggieri M, Fumarola A, et al. A mathematical formula to estimate in vivo thyroid volume from two-dimensional ultrasonography. Thyroid 18: 879-882, 2008.

9. Kennedy RL, Griffiths H, Gray TA. Amiodarone and the thyroid. Clin Chem 35: 1882-1887, 1989.

10. Bogazzi F, Tomisti L, Bartalena L, Aghini-Lombardi F, Martino E. Amiodarone and the thyroid: a 2012 update. J Endocrinol Invest 35: 340-348, 2012.

11. Eskes SA, Endert E, Fliers E, et al. Treatment of amiodaroneinduced thyrotoxicosis type 2: a randomized clinical trial. J Clin Endocrinol Metab 97: 499-506, 2012.

12. Bogazzi F, Tomisti L, Rossi G, et al. Glucocorticoids are preferable to thionamides as first-line treatment for amiodarone-induced thyrotoxicosis due to destructive thyroiditis: a matched retrospec- tive cohort study. J Clin Endocrinol Metab 94: 3757-3762, 2009.

13. Bogazzi F, Bartalena L, Tomisti L, et al. Glucocorticoid response in amiodarone-induced thyrotoxicosis resulting from destructive thyroiditis is predicted by thyroid volume and serum free thyroid hormone concentrations. J Clin Endocrinol Metab 92: 556-562, 2007.

14. Gough J, Gough IR. Total thyroidectomy for amiodaroneassociated thyrotoxicosis in patients with severe cardiac disease. World J Surg 30: 1957-1961, 2006.

15. Smyrk TC, Goellner JR, Brennan MD, Carney JA. Pathology of the thyroid in amiodarone-associated thyrotoxicosis. Am J Surg Pathol 11: 197-204, 1987.

16. Nakazawa T, Murata S, Kondo T, et al. Histopathology of the thyroid in amiodarone-induced hypothyroidism. Pathol Int 58: 55-58, 2008.

17. Ando T, Henmi T, Haruta D, et al. Graves' disease complicated by ventricular fibrillation in three men who were smokers. Thyroid 21: 1021-1025, 2011.

18. Brooks MJ, Pattison DA, Teo EP, Price S, Gurvitch R. Amiodarone-induced destructive thyroiditis associated with coronary artery vasospasm and recurrent ventricular fibrillation. Eur Thyroid J 2: 65-67, 2013.

19. Kung AW. Clinical review: thyrotoxic periodic paralysis: a diagnostic challenge. J Clin Endocrinol Metab 91: 2490-2495, 2006.

20. Abbasi B, Sharif Z, Sprabery LR. Hypokalemic thyrotoxic periodic paralysis with thyrotoxic psychosis and hypercapnic respiratory failure. Am J Med Sci 340: 147-153, 2010.

21. Wu CZ, Wu YK, Lin JD, Kuo SW. Thyrotoxic periodic paralysis complicated by acute hypercapnic respiratory failure and ventricular tachycardia. Thyroid 18: 1321-1324, 2008.

The Internal Medicine is an Open Access article distributed under the Creative Commons Attribution-NonCommercial-NoDerivatives 4.0 International License. To view the details of this license, please visit (https://creativecommons.org/licenses/ by-nc-nd/4.0/).

(C) 2018 The Japanese Society of Internal Medicine

Intern Med 57: 59-63, 2018 